\title{
Production of Macrophomina phaseolina Conidia by Multiple Soybean Isolates in Culture
}

\author{
J. Ma and C. B. Hill, Department of Crop Sciences, University of Illinois at Urbana-Champaign, Urbana 61801; and \\ G. L. Hartman, United States Department of Agriculture-Agricultural Research Services and Department of Crop \\ Sciences, University of Illinois at Urbana-Champaign, Urbana 61801
}

\begin{abstract}
Ma, J., Hill, C. B., and Hartman, G. L. 2010. Production of Macrophomina phaseolina conidia by multiple soybean isolates in culture. Plant Dis. 94:1088-1092.

Macrophomina phaseolina is the cause of charcoal rot of soybean (Glycine max). Resistance to M. phaseolina in commercial soybean cultivars is not common but is needed in locations where the disease is chronic and severe. The objective of this study was to develop a reliable method to produce sufficient $M$. phaseolina conidia that can be used to inoculate soybean plants in a highthroughput resistance-screening program. Production of pycnidia is not common on most culture media, such as potato dextrose agar, but can be produced on media containing plant parts or oilseed extracts. Seven semi-defined media were tested to induce pycnidia production. Results indicated that the number of pycnidia that were produced by eight $M$. phaseolina isolates was dependent on induction medium; however, peanut butter extract-saturated filter paper placed over soynut butter extract agar (PESEA) allowed for greater pycnidia and conidia production than the other media tested. Production of pycnidia on PESEA ranged from 269 to 1,082 per plate. There were no differences among isolates in germination of conidia produced on PESEA, which averaged $83 \pm 2 \%$ germination. A conidial suspension from one $M$. phaseolina isolate produced on PESEA and inoculated onto soybean radicles significantly distinguished $(P<0.01)$ 'DT97-4290', a soybean genotype with partial resistance to charcoal rot, from a susceptible genotype, 'LS98-0358'. Results of this study indicated that multiple isolates of M. phaseolina from soybean produced sufficient amounts of conidia on PESEA to use as inoculum. This conidia inoculum production method will facilitate soybean charcoal rot resistance screening evaluation with different soybean isolates.
\end{abstract}

Macrophomina phaseolina (Tassi) Goid., a pycnidia-producing fungus, is one of the most important soilborne pathogens, infecting over 500 plant species in more than 100 plant families around the world $(1,18)$. It causes a major disease of soybean (Glycine $\max (\mathrm{L}$.) Merr.) called charcoal rot (18). Symptoms on soybean include blight of emerging seedlings, wilting, early maturation, and incomplete pod filling (18). The disease is most pronounced when plants are stressed by adverse environ-

Corresponding author: G. L. Hartman

E-mail: ghartman@illinois.edu

Trade and manufacturers' names are necessary to report factually on available data; however, the USDA neither guarantees nor warrants the standard of the product, and the use of the name by USDA implies no approval of the product to the exclusion of others that may also be suitable.

Accepted for publication 27 April 2010.

doi:10.1094/PDIS-94-9-1088

This article is in the public domain and not copyrightable. It may be freely reprinted with customary crediting of the source. The American Phytopathological Society, 2010. mental factors such as drought and heat stress during the reproductive growth stages. Charcoal rot of soybean occurs in all soybean production areas throughout the world (18).

Diagnostic signs of charcoal rot are the microsclerotia that are often imbedded in infected host debris. The pycnidial stage is commonly found in nature on garden bean and jute bean (18), occasionally on soybean and other host plants (1), and has even been reported on cats (5). Pycnidia have been reported to develop on agar media such as potato dextrose agar (PDA) $(3,20)$ and soybean meal agar $(10)$, although infrequently. In vitro production of large numbers of pycnidia has been accomplished using sterilized plant parts, filter paper treated with peanut meal-ether extract placed on the surface of a basal agar medium, and incubation under UV and long-wave near-UV irradiation, with variable results depending on $M$. phaseolina isolate and substrate $(1,2,4,7-11,13)$.

Various kinds of inocula have been used to inoculate plants with $M$. phaseolina, including microsclerotia suspensions, a mixture of sclerotia and soil, or infested millet $(12,18,19)$; however, there are no reports of the use of a conidia suspension as inoculum, which has the advantage that it can be easily quantified with precision and then standardized based on the number of conidia per unit of inoculum. Standardized inoculum is essential for sound comparative studies (2), especially when differences between treatments, such as between partially resistant host plant genotypes, are relatively small. Standardizing inoculum and inoculation procedures also maximizes reliability and repeatability of inoculations. A method to produce abundant M. phaseolina conidia from different soybean isolates to use as inoculum would facilitate screening for partial resistance among soybean genotypes and, possibly, also could be used with other crop hosts. $(1,2,4,7-11)$. The objective of this study was to select a method that would induce multiple soybean isolates to produce a sufficient quantity of viable and infective $M$. phaseolina conidia that could be used to inoculate soybean plants in a large resistance-screening program.

\section{MATERIALS AND METHODS}

M. phaseolina isolates. Eight $M$. phaseolina isolates collected from soybean were tested in this study (Table 1). The isolates were maintained on PDA (Difco Laboratories, Sparks, MD) at $28^{\circ} \mathrm{C}$ and were periodically passed through plants to maintain aggressiveness.

Pycnidial induction media. Two experiments were conducted. In experiment 1 , seven different semi-defined media were tested for the ability to induce $M$. phaseolina pycnidia production. These test media were selected for further testing after trying several of the methods listed in Dhingra and Sinclair (1), with some modifications, in a number of preliminary experiments. Instructions for preparing each medium are described below. All media were sterilized by autoclaving for $20 \mathrm{~min}$ at $121^{\circ} \mathrm{C}$ and 115 psi. Each medium $(20 \mathrm{ml})$ was poured into $9-\mathrm{cm}$ diameter sterile plastic petri dishes after cooling to $45^{\circ} \mathrm{C}$. Following solidification of the agar media, a single Whatman no. 1 filter paper disk ( $4.5 \mathrm{~cm}$ in diameter), previously sterilized by autoclaving for 15 min at $121^{\circ} \mathrm{C}$ and $115 \mathrm{psi}$, and with or 
without additional treatment, was placed on the surface of each plate.

The first test medium was untreated sterile filter paper placed on the top of $2 \%$ water agar (WA), and was referred to as FWA. The WA was prepared by adding 20 $\mathrm{g}$ of Bacto agar to 1 liter of distilled water. This medium served as a control since the filter paper was not treated and the agar media was WA and not PDA.

A second test medium (FPDA) was prepared from dehydrated PDA (Difco Laboratories) according to label directions. A plain, untreated, sterile filter paper disk was placed in each plate. This medium served as a control for the effects of untreated filter paper and agar media with different nutrient composition.

The third tested medium, peanut butter extract agar (PEA), was prepared as follows: peanut butter extract (PE) was prepared by combining $20 \mathrm{~g}$ of peanut butter (East Wind Community, Inc., Tecumseh, MO) and $100 \mathrm{ml}$ of ether into a $500-\mathrm{ml}$ storage bottle. The mixture was shaken by hand until the peanut butter dissolved in the ether, and then allowed to stand in a chemical fume hood for at least $12 \mathrm{~h}$ to separate. The supernatant was decanted into a new flask and placed, uncapped, onto an orbital shaker inside the fume hood until the ether evaporated, leaving the PE in the flask. Adding $500 \mathrm{ml}$ of distilled water and $7.5 \mathrm{~g}$ of Bacto agar to the PE made PEA.

A fourth medium tested, soynut butter extract agar (SEA), was prepared with an ether extract from soynut butter (SE), using the method similar to the PE except with $6 \mathrm{~g}$ of soynut butter (The SoyNut Butter Company, Glenview, IL) added to $100 \mathrm{ml}$ of ether to make the extract used in the medium, followed by adding $500 \mathrm{ml}$ of distilled water and $7.5 \mathrm{~g}$ of Bacto agar to the PE.

The fifth medium tested was PE-treated filter paper on the top of soynut extract agar (PESEA) that was prepared from PE and SEA. Filter paper disks were treated with PE by soaking 50 filter paper disks in $100 \mathrm{ml}$ of PE supernatant until the ether evaporated, leaving the $\mathrm{PE}$ on the filter paper disks. After plates containing SEA cooled, a single autoclaved PE-treated filter paper disk was placed on the surface of each SEA plate to make the PESEA medium.

A sixth medium tested consisted of refined expeller-pressed canola oil (Hain Celestial Group, Inc., Boulder. Co.)-treated filter paper on the top of SEA (COSEA). The canola-oil-treated filter paper was prepared by dipping the filter paper into canola oil prior to autoclaving. One treated filter paper disk was placed on the surface of each SEA plate.

The seventh medium tested was refined cold pressed olive oil (Hain Celestial Group, Inc.)-treated filter paper placed on top of SEA (OOSEA). Olive-oil-treated filter paper was prepared similarly to the canola oil filter paper by dipping the filter paper into olive oil prior to autoclaving.

A follow-up experiment (experiment 2) duction on the semi-defined medium that appeared to have the highest potential to induce pycnidia development, based on the results of experiment 1 , with production on plain, sterile soybean leaflets referred to as leaf water agar (LWA). Soybean leaflets after maximum expansion were collected from the youngest trifoliolates of 21- to 28-day-old plants of soybean cv. Williams 82 grown in a plant growth chamber and allowed to wilt for $24 \mathrm{~h}$. The wilted leaflets were surface disinfected with $75 \%$ ethyl alcohol for $2 \mathrm{~min}$ and $0.5 \% \mathrm{NaOCl}$ for 5 min, followed by rinsing in sterile, distilled water twice. A single leaflet was placed inside each 9-cm-diameter sterile plastic petri dish containing $20 \mathrm{ml}$ of sterile, $1 \%$ water agar (10 $\mathrm{g}$ of Bacto agar per liter of distilled water).

The eight M. phaseolina isolates were transferred from PDA maintenance cultures to fresh PDA plates and incubated at $28^{\circ} \mathrm{C}$ in the dark for 4 to 5 days. Agar plugs ( $4 \mathrm{~mm}$ in diameter) were cut with a sterile cork borer from the margin of an actively growing PDA culture. A single agar plug with $M$. phaseolina mycelium was placed in the center of each test plate. All plates were then sealed with a single layer of Parafilm (Pechiney Plastic Packaging, Chicago), incubated in the dark at $28^{\circ} \mathrm{C}$ for $24 \mathrm{~h}$, and then further incubated at $22^{\circ} \mathrm{C}$ for 7 to 10 days at a distance of about $20 \mathrm{~cm}$ below continuous 20 -W black light blue (BLB) fluorescent lamps (Phillips Lighting Co., Somerset, NJ).

Experimental designs and data collected. Both experiments were set up as factorials with the eight $M$. phaseolina isolates as one factor and the pycnidial induction media as a second factor. The factor combinations in each test were arranged in a completely randomized design with three replications. In experiment 1 , the days to appearance of the first pycnidium, defined in this study as having visible conidial ooze extruding from the ostiole, was recorded. In addition, the number of pycnidia per plate, an estimate of number of conidia produced per plate, was conducted to compare pycnidial pro-

and the percent conidia germination were recorded. In experiment 2 , the number of pycnidia per plate and conidia produced per 80 pycnidia were recorded, and conidia collected in this experiment were also tested for infectivity on soybean radicles.

Pycnidial enumeration and measurement of pycnidia dimensions. Pycnidia in each plate were visually counted with the aid of an Olympus SZX16 stereomicroscope (Olympus America, Inc., Center Valley, PA) at $\times 50$ magnification. The total number of pycnidia per plate was the experimental unit for statistical analysis.

Widths and lengths in micrometers of nine randomly chosen pycnidia in experiment 2 for each isolate-media combination were also measured. The widest part of each pycnidium and the length from the top of the ostiole to the base of the pycnidium were measured with the use of an Olympus BX51 compound microscope equipped with a precalibrated ocular micrometer at $\times 200$ magnification. In addition, using the same microscope at $\times 400$, 40 conidial lengths and widths were measured from four isolates (MP3, TN4, TN5, and TN146) produced on PESEA.

Quantification of conidia production. All mature pycnidia, with oozing conidia, on the plates of each $M$. phaseolina isolate on each induction medium in each replication in experiment 1 and 80 mature pycnidia from each plate in experiment 2 were placed into tubes with distilled $\mathrm{H}_{2} \mathrm{O}$ and shaken on a Vortex mixer until the pycnidia were broken open, releasing the conidia. The number of conidia in each plate was estimated from conidia counts in three independent samples with the aid of a hemacytometer.

Conidia germination and infectivity. The percent germination and infectivity of conidia of each isolate harvested from PESEA in experiment 2 was tested. Two soybean genotypes, 'DT97-4290', partially resistant to charcoal rot $(12,15)$, and 'LS98-0358', a charcoal-rot-susceptible genotype obtained from J. Bond, Southern Illinois University, Carbondale, were selected to test the infectivity of conidia from the eight $M$. phaseolina isolates produced on PESEA in experiment 2. Seed were surface disinfected with $1 \% \mathrm{NaOCl}$ for $8 \mathrm{~min}$; rinsed twice with sterile, dis-

Table 1. Macrophomina phaseolina isolates from soybean evaluated for conidial production

\begin{tabular}{llll}
\hline Isolate & Origin & Year & Source \\
\hline TN4 & Jackson, TN & 2006 & A. Mengistu \\
TN5 & Ames, TN & 2006 & A. Mengistu \\
TN146 & Neosho, TN & 2006 & A. Mengistu \\
Mp2003 & Urbana, IL & 2003 & G. Hartman \\
Mp3 & Champaign, IL & 1998 & J. Manandahary \\
Conway & Conway, AR & 2004 & J. Rupe \\
Kibler & Kibler, AR & 2008 & J. Rupe \\
Pinetree & Pinetree, AR & 1998 & J. Rupe \\
\hline
\end{tabular}

x United States Department of Agriculture-Agricultural Research Service, Jackson, TN.

y Dairyland Seed Company, Gibson City, IL.

${ }^{\mathrm{z}}$ University of Arkansas, Fayetteville, AR. 
tilled water; plated onto PDA; and incubated at $26^{\circ} \mathrm{C}$ for $24 \mathrm{~h}$. Seed visually free of microbial infection were transferred to fresh $1.5 \%$ WA plates and incubated at $26^{\circ} \mathrm{C}$ for another $48 \mathrm{~h}$ until seedling radicles grew to 1 to $2 \mathrm{~cm}$ long.

Conidia from each isolate for inoculation was collected by first disrupting the pycnidia on the filter paper disks with a sterile scalpel blade, followed by washing with $15 \mathrm{ml}$ of sterile $0.05 \%$ Tween-20 solution to remove the conidia released from the pycnidia. The conidia suspension was filtered with a sterile $37-\mu \mathrm{m}$ nylon mesh (Small Parts, Inc., Miramar, FL) to remove pycnidial debris and mycelia. The percent germination of three independent $10-\mu \mathrm{l}$ samples from the conidial suspension of each replication was recorded after $8 \mathrm{~h}$ of incubation on glass slides at $28^{\circ} \mathrm{C}$ and $100 \%$ relative humidity in the dark by counting germinated or not germinated conidia with the aid of the Olympus BX51 compound microscope at $\times 40$ magnification. Conidia from the Pinetree, AR isolate was used to test the infectivity of the conidia on soybean radicles because preliminary tests indicated that it was the most aggressive isolate. Pinetree conidia were standardized to 250 conidia/ $\mu$ with the aid of a hemacytometer. Soybean radicles were inoculated with a 2- $\mu$ l droplet deposited in the center of each radicle with a pipette. After 3 days of incubation in the dark at $28^{\circ} \mathrm{C}$, the length of each lesion (millimeters) produced by $M$. phaseolina infection was measured. The lesion length from 39 radicles of each soybean genotype inoculated with the Pinetree isolate was recorded.
Statistical analysis. Analysis of variance (ANOVA) and means comparisons were performed with the aid of JMP, version 5. The number of pycnidia and conidia was transformed by adding 1 and then taking the logarithm to the base 10 prior to analysis to correct for unequal variance and experimental units without pycnidia. Means of factor levels (isolates and pycnidial induction media) or interactions between factors for each parameter were separated using the least significant difference at $P=0.05$ when the ANOVA indicated significance of differences among them.

\section{RESULTS}

Comparison of pycnidial and conidial production on seven pycnidia induction media in experiment 1. There was a highly significant $(P<0.01)$ effect of media on days to first pycnidium, primarily due to the effect of PESEA (6.0 days to first pycnidium) that had significantly lower $(P<0.05)$ days to first pycnidium than OOSEA (7.8 days), COSEA (8.3 days), SEA (8.7 days), and PEA (9.3 days), which did not differ significantly. No pycnidia were formed on FWA or FPDA. Differences among isolates and the interaction of medium-isolate were nonsignificant $(P>0.05)$.

There was a highly significant $(P<$ 0.01 ) interaction between $M$. phaseolina isolates and pycnidial induction media for number of pycnidia per plate (Fig. 1), indicating that the number of pycnidia produced by each $M$. phaseolina isolate depended on the induction medium. The

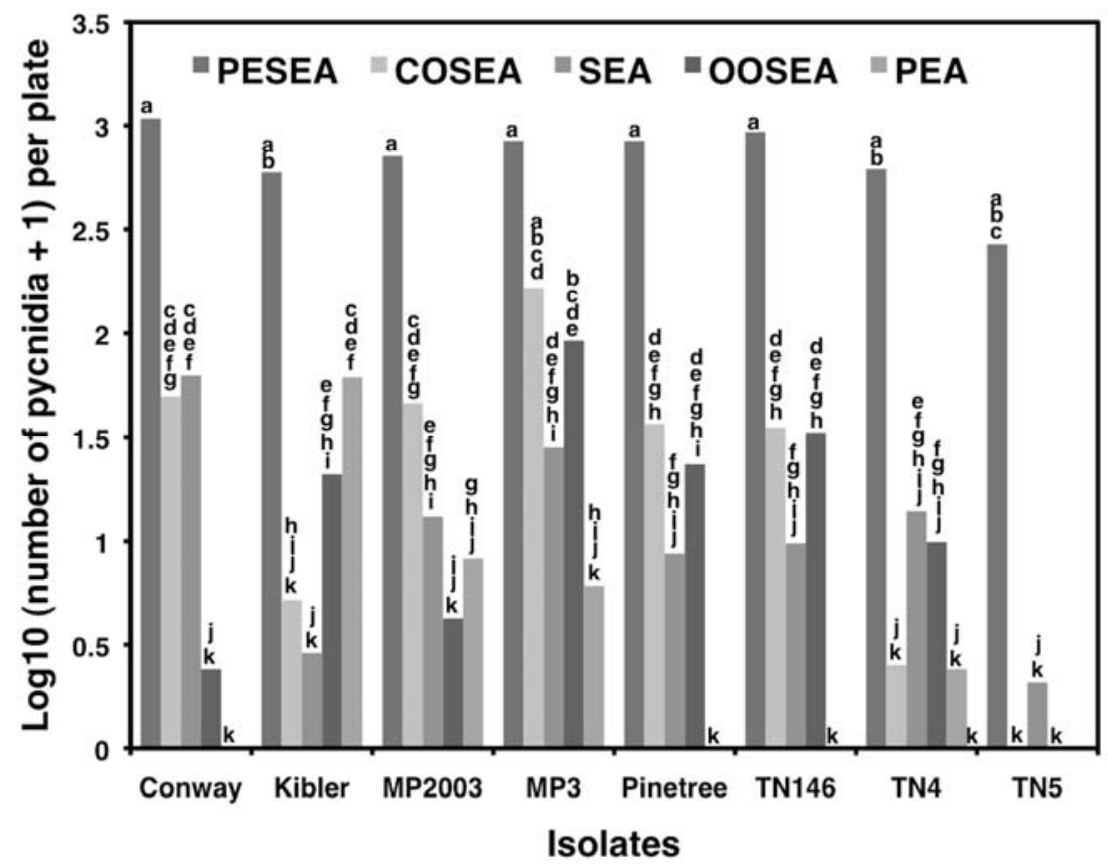

Fig. 1. Number of pycnidia produced per plate by eight Macrophomina phaseolina isolates on five media: peanut extract-treated filter paper on the top of soynut butter extract agar (SEA) (PESEA), canola oil-treated filter paper on the top of SEA (COSEA), SEA, olive oil-treated filter paper on the top of SEA (OOSEA), and peanut butter extract agar (PEA). Bars with the same letter are not significantly different at $P=0.05$. highest number of pycnidia per plate was produced by the isolate Conway on PESEA (1,072 pycnidia/plate), and PESEA medium had the highest pycnidial production for each isolate compared with the other media, except with isolate $\mathrm{Mp} 3$, which had pycnidial production on COSEA that was not significantly different than production on PESEA. The number of pycnidia produced on COSEA was similar to production on OOSEA, whereas pycnidial production on SEA tended to be higher than on PEA. The isolate Mp3 tended to produce more and TN5 less pycnidia over all of the semi-defined media than the other isolates.

There was also a highly significant interaction $(P<0.01)$ between $M$. phaseolina isolates and pycnidial induction media for conidia produced per plate (Fig. 2), indicating the dependence of each isolate on induction medium for the amount of conidia produced. Isolate Mp3 on PESEA had the highest conidia per plate $\left(2.1 \times 10^{5}\right.$ conidia/plate) but the amount was not significantly higher $(P>0.05)$ than the amounts on COSEA, SEA, or OOSEA. As with pycnidial production, the amount of conidia produced by each isolate was higher on PESEA than on the other media. Conidia production was similar on COSEA and OOSEA, SEA tended to produce more conidia than PEA, and there were no pycnidia (thus, no conidia) produced on plain filter paper placed on the top of WA or PDA.

Production of pycnidia and conidia of eight isolates on PESEA and LWA. A highly significant $(P<0.01)$ interaction was found between the two pycnidial induction media, PESEA and LWA, and eight $M$. phaseolina isolates for pycnidia produced per plate in experiment 2 (Fig. $3)$, indicating the dependence between media and isolate as found in experiment 1. Pycnidial production by the isolates was similar on both media except for the isolate Conway, which had a significantly higher number of pycnidia per plate on PESEA (924 pycnidia/plate) than on LWA (506 pycnidia/plate). The Kibler isolate on LWA had the highest number of pycnidia per plate (956 pycnidia/plate) but it was not significantly $(P>0.05)$ higher than its production on PESEA (799 pycnidia/ plate).

There were highly significant $(P<0.05)$ differences between the main effects, induction media (PESEA and LWA), and isolates for the amount of conidia produced per 80 pycnidia; however, no significant interaction occurred between these main effects. LWA produced $2.6 \times 10^{4}$ and PESEA produced $2.3 \times 10^{4}$ conidia per 80 pycnidia. Isolate Mp2003 produced significantly $(P<0.05)$ more conidia than all other isolates, and Conway produced the lowest number of conidia per 80 pycnidia, which was significantly $(P<0.05)$ lower than all of the other isolates (Table 2). 
Germination and infectivity of conidia produced on PESEA. There were no significant differences among the isolates for percent germination of conidia produced on PESEA. The mean percent germination was $83 \pm 2 \%$. All 39 radicles of each inoculated genotype inoculated with a conidial suspension produced a visible necrotic lesion. Lesion length produced on the charcoal-rot-susceptible soybean line
LS98-0358 (2.9 mm) was significantly higher $(P<0.01)$ than the lesion length on the partially resistant cv. DT97-4290 (0.5 $\mathrm{mm}$ ), indicating that the Pinetree conidia were highly infective and could be used to differentiate a susceptible from a partially resistant genotype.

Morphological characteristics of $M$. phaseolina pycnidia and conidia. $M$. phaseolina pycnidial and conidial dimen-

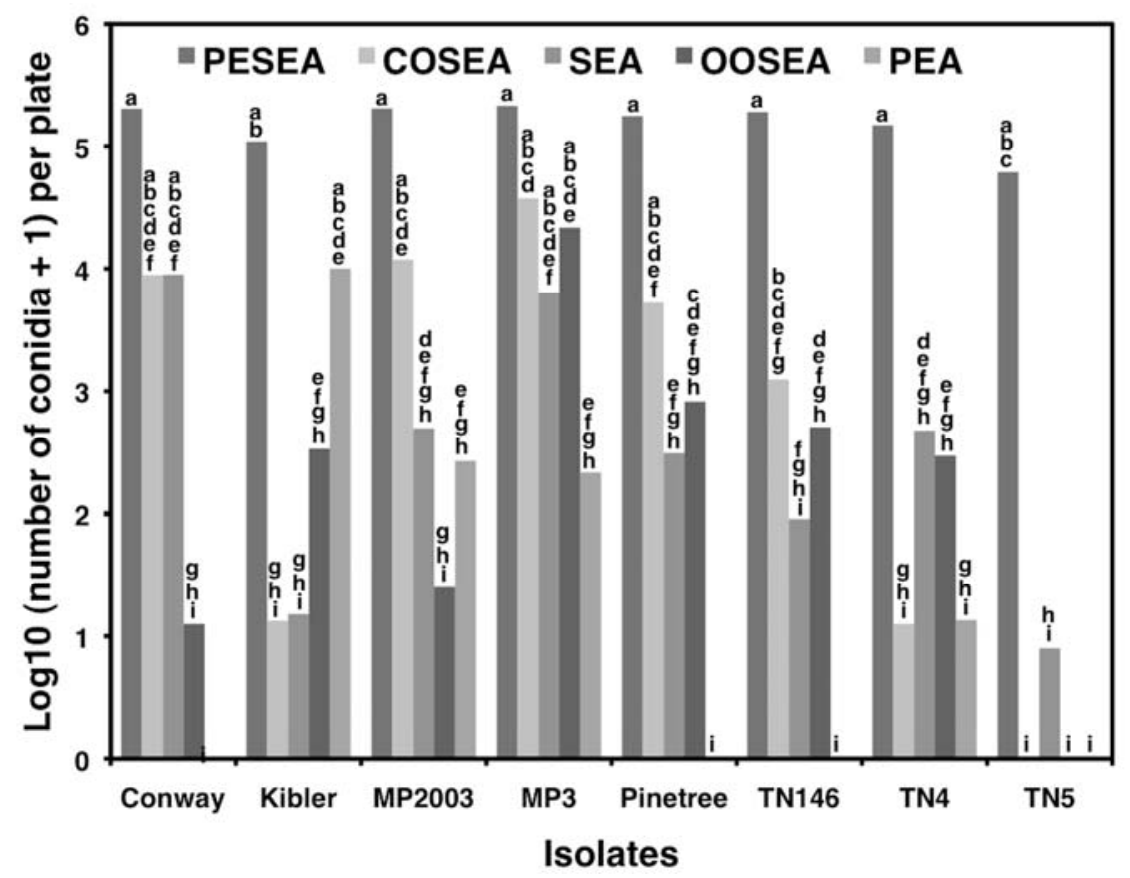

Fig. 2. Number of conidia produced per plate by eight Macrophomina phaseolina isolates on five media: peanut extract-treated filter paper on the top of soynut butter extract agar (SEA) (PESEA), canola oil-treated filter paper on the top of SEA (COSEA), SEA, olive oil-treated filter paper on the top of SEA (OOSEA), and peanut butter extract agar (PEA). Bars with the same letter are not significantly different at $P=0.05$.

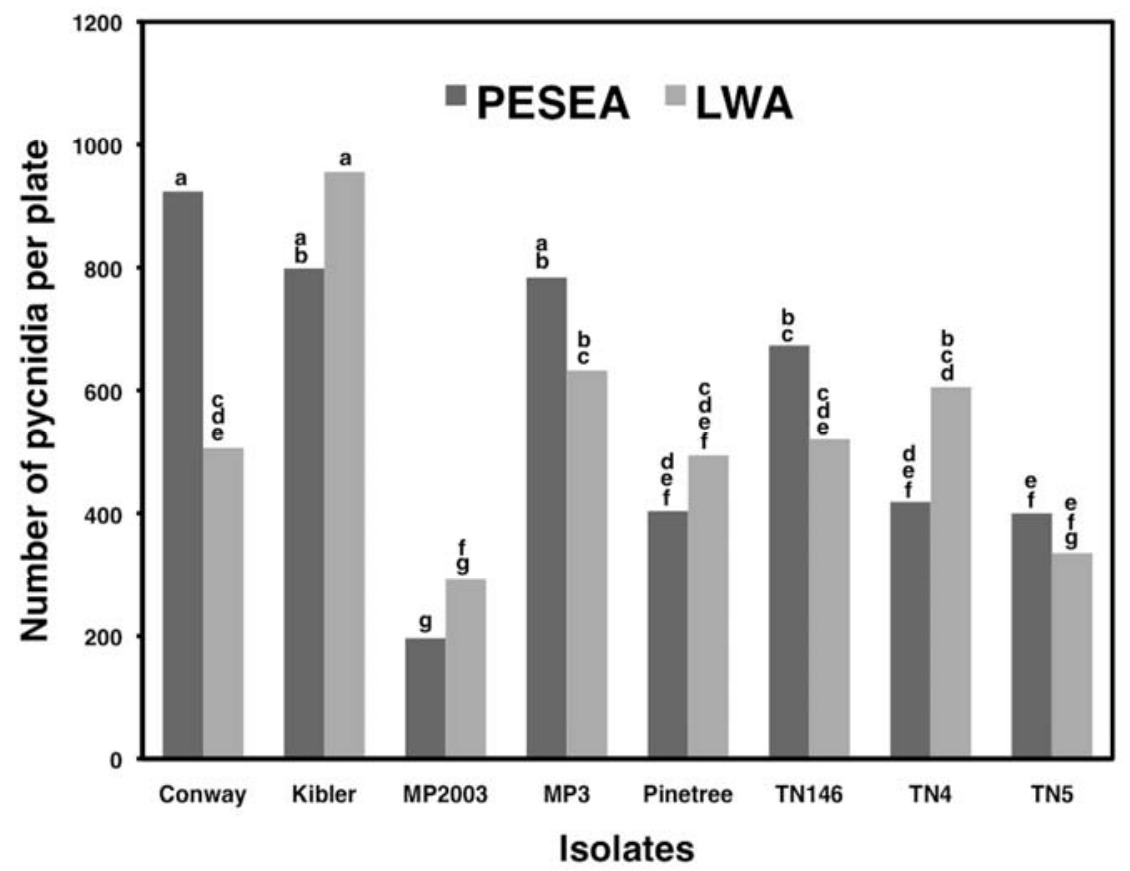

Fig. 3. Number of pycnidia produced by eight Macrophomina phaseolina isolates on leaf water agar (LWA) and peanut extract-treated filter paper on the top of soynut butter extract agar (PESEA). Bars with the same letter are not significantly different at $P=0.05$. sions fit the descriptions reported by the species $(1,4,10)$. Pycnidia were globose and erumpent at maturity on soybean leaflets. On filter paper, the pycnidia were globose to fusiform. All pycnidia observed were dark to grayish colored and each appeared to have a conspicuous, single, clear or hyaline ostiole. Elliptical, unicellular conidia oozed from the ostioles of mature pycnidia, with dimensions in a 3:1 length/width ratio, in agreement with previous reports (1).

\section{DISCUSSION}

The quality and quantity of pathogen inoculum influence host reaction during disease establishment (2). High repeatability of inoculations is enhanced by standardization of both inoculum quality and quantity (2). Previous soybean charcoal rot resistance tests involved inoculating soybean plants with infested grain, such as millet (19), or planting in infested soil $(12,15)$. Although these methods have been suitable for comparing the resistance in a relatively few soybean genotypes in an experiment (19), they may not be practical in large experiments with a number of genotypes. For example, inoculum and inoculation standardization is critical to determine resistance phenotypes in tests of mapping populations derived from crosses between resistant and susceptible genotypes, where several hundred plants may need to be tested with high repeatability to detect small differences in resistance.

With improving repeatability of $M$. phaseolina inoculations a goal, the primary objective of this study was to find a method that produced sufficiently abundant $M$. phaseolina conidia from different soybean isolates to use as inoculum to efficiently screen a large number of soybean genotypes for resistance to charcoal rot. Various culture media have been used to induce $M$. phaseolina pycnidia production (1,2,7-9). We had previously screened over 20 different media previously reported $(1,2,8,9)$ for production of conidia. Based on those preliminary studies, three media, PEA, SEA, and soybean leaflets,

Table 2. Mean number of conidia produced in 80 pycnidia formed on leaf water agar and on peanut extract-treated filter paper on soynut butter extract agar by eight Macrophomina phaseolina isolates ${ }^{\mathrm{z}}$

\begin{tabular}{lc}
\hline Isolate & No. of conidia $\times \mathbf{1 0}^{\mathbf{4}}$ \\
\hline MP2003 & $4.3 \mathrm{a}$ \\
Pinetree & $3.7 \mathrm{~b}$ \\
MP3 & $3.1 \mathrm{c}$ \\
TN4 & $2.7 \mathrm{~cd}$ \\
TN146 & $2.3 \mathrm{~d}$ \\
Kibler & $1.6 \mathrm{e}$ \\
TN5 & $1.3 \mathrm{e}$ \\
Conway & $0.6 \mathrm{f}$ \\
\hline
\end{tabular}

$\mathrm{z}$ There was no significant interaction of mediaisolate; thus, means were averaged over the two media. Means followed by the same letter were not significantly different at $P=0.05$. 
appeared to be the most promising; thus, they were examined in this study. PEA and SEA were compared with four other media and the most promising was compared with soybean leaflets. Although pycnidia production of eight $M$. phaseolina isolates was dependent on the induction medium in this study, overall, PESEA and soybean leaflets provided for good levels of pycnidia and conidia production by each of the soybean isolates tested.

PESEA is a modification of the method reported by Knox-Davies $(8,9)$, with differences in the agar medium supporting the PE-treated filter-paper disk and the quality of irradiation during incubation. KnoxDavies used a more defined medium containing $\mathrm{MgSO}_{4}, \mathrm{KH}_{2} \mathrm{HPO}_{4}$, glucose, peptone, and DL-asparagine than used in our study. In addition, cultures were incubated first for $24 \mathrm{~h}$ in the dark, followed by long wave UV light for up to 5 days, with a minimum of $24 \mathrm{~h}$ needed to induce sporulation (9). Our tests indicated that near-UV irradiation, provided by the BLB fluorescent tubes, was sufficient to induce pycnidial production on PESEA and other media tested.

No pycnidia were produced on plain filter paper placed on the top of WA or PDA, indicating that substances extracted from soynut and peanut butter or from canola or olive oil were helpful in inducing pycnidia formation. Oleic acid in ether extracts from peanut was previously found to encourage $M$. phaseolina pycnidia production under UV irradiation (14), and this was corroborated in this study. Although olive and canola oils contain higher levels of oleic acid than peanut (6), in this study, the PE-treated filter paper tended to produce more pycnidia overall. Therefore, it appears that there may be other substances in the PE that helped encourage pycnidia production. The number of pycnidia produced on either PEA and SEA without filter paper was relatively low compared with PESEA. This suggested that the filter paper provided a platform similar to the highly cellulosic plant debris on which pycnidia are produced in nature. Filter paper was previously reported to encourage $M$. phaseolina pycnidia production (17) and sporulation in other imperfect fungi (16).

Although there were no significant differences in pycnidial induction between
LWA and PESEA, conidia were much more easily harvested and had less debris when harvested from filter paper compared with leaves. In addition, it is possible that leaflets from other soybean genotypes, developmental stages, or ages could give different results than those found in this study. Therefore, the semi-defined PESEA medium may produce more consistent results in different laboratories.

The pycnidial stage of $M$. phaseolina is common on garden and jute bean cultivars and has also been reported on soybean (8). Pycnidia immersed in infected host tissues may provide conidial inoculum for secondary spread among host plants in the field, although this does not appear to be common, at least in soybean grown in the United States where pycnidial occurrence on soybean debris appears to be infrequent. The use of $M$. phaseolina conidia to inoculate plants for resistance evaluation had not been previously reported, possibly because previous attempts to produce pycnidia in culture were not inconsistent. Results in this study demonstrated that $M$. phaseolina pycnidia from multiple soybean isolates were produced in culture and that inoculation with conidial inoculum can distinguish charcoal-rot-resistant from -susceptible soybean genotypes. Use of PESEA to produce conidia for inoculum for use in a high-throughput charcoal rot resistance screening program will greatly facilitate charcoal rot resistance evaluation and could help expedite development of new soybean cultivars with improved charcoal rot resistance.

\section{ACKNOWLEDGMENTS}

We thank the United Soybean Board and the North Central Soybean Research Program for partial support of this research.

\section{LITERATURE CITED}

1. Dhingra, O. D., and Sinclair, J. B. 1978. Biology and Pathology of Macrophomina phaseolina. Universidade Federal de Viçosa, Viçosa.

2. Dhingra, O. D., and Sinclair, J. B. 1995. Basic Plant Pathology Methods. CRC Press, Boca Raton, FL.

3. Gaetán, S. A., Fernandez, L., and Madia, M. 2006. Occurrence of charcoal rot caused by Macrophomina phaseolina on Canola in Argentina. Plant Dis. 90:524.

4. Goth, R., and Ostazeshi, S. 1965. Sporulation of Macrophomina phaseoli on propylene oxide-sterilized leaf tissue. Phytopathology 55:1156.

5. Hasegawa, T., Yoshida, Y., Kosuge, J., Haga, T., Goto, Y., Shinjo, T., Uchida, K., Yama- guchi, R., Tateyama, S., and Takatori, K. 2005. Subcutaneous granuloma associated with Macrophomina species infection in a cat. Vet. Rec. 156:23-24.

6. Hilditch, T. 2007. The chemical constitution of natural fats. Br. J. Nutr. 3:347-354

7. Hussain, M., and Ahmed, Q. 1960. Studies on the sporulation of Macrophomina phasioli (Maubl.) Ashby., causing stem-rot disease of jute with reference to the possible causes of outbreak of the disease in nature. J. Pak. Sci. Ind. Res. 3:219-225.

8. Knox-Davies, P. S. 1965. Pycnidium production by Macrophomina phaseoli. S. Afr. J. Agric. Sci. 8:205-218.

9. Knox-Davies, P. S. 1966. Further studies on pycnidia production by Macrophomina phaseoli. S. Afr. J. Agric. Sci. 9:595-600.

10. Machado, C. 1987. Macrophomina phaseolina: biological behavior of isolates, spatial pattern of microsclerotia in the soil, and incidence on soybeans. Ph.D. diss. University of Illinois, Urbana.

11. McCain, A. H., and Scharpf, R. F. 1989. Effect of inoculum density of Macrophomina phaseolina on seedling susceptibility of six conifer species. Eur. J. Forest Pathol. 19:119-123.

12. Mengistu, A., Ray, J. D., Smth, G. D., and Paris, R. L. 2007. Charcoal rot disease assessment of soybean genotypes using colonyforming units index. Crop Sci. 47:2453-2461.

13. Mihail, J., and Taylor, S. 1995. Interpreting variability among isolates of Macrophomina phaseolina in pathogenicity, pycnidium production, and chlorate utilization. Can. J. Bot. 73:1596-1603.

14. Oosthuizen, M. M. J., and Potgieter, D. J. J. 1974. Induction of photosporogenesis in Macrophomina phaseoli by an octadecenoic acid from peanuts. Phytochemistry 13:1027-1029.

15. Paris, R. L., Mengistu, A., Tyler, J. M., and Smith, J. R. 2006. Registration of soybean germplasm line DT97-4290 with moderate resistance to charcoal rot. Crop Sci. 46:2324-2325.

16. Pratt, R. G. 2006. Enhancement of sporulation in species of Bipolaris, Curvularia, Drechslera, and Exserohilum by growth on cellulosecontaining substrates. Mycopathologicia 162: 133-140.

17. Rodrigues, V. J. L. B., Menezes, M., and Coelho, R. S. B. 1997. Efeito da temperature, papel de filtro e meio de cultura na fisiologia de Macrophomina phaseolina. Arq. Biol. Tecnol. Curitiba 40:197-203.

18. Smith, G. S., and Wyllie, T. D. 1999. Charcoal rot. Pages 29-31 in: Compendium of Soybean Diseases. G. L. Hartman, J. B. Sinclair, and J. C. Rupe, eds. American Phytopathological Society, St. Paul, MN.

19. Srivastava, A. K., and Singh, B. B. 1990. Effect of organic amendment on interaction of Macrophomina phaseolina and Meloidogyne incognita on fresh beans (Phaseolus vulgaris). New Agric. 1:99-100.

20. Yang, X. B., and Navi, S. S. 2005. First report of charcoal rot epidemics caused by Macrophomina phaseolina in soybean in Iowa. Plant Dis. 89:526. 